\title{
The Relationship between the Care Burden, Anxiety, and Social Support Levels of Mothers of Children with Cancer
}

\author{
(D) Esra Nur KOCAASLAN, (D) Melahat AKGÜN KOSTAK, (i) Remziye SEMERCi, (1) İsmail ÇETiNTAŞ \\ Department of Nursing, Trakya University, Edirne-Turkey
}

\begin{abstract}
OBJECTIVE
This descriptive study was conducted to determine the relationship between the care burden, anxiety, and social support levels of the mothers who had children with cancer and affecting factors.

\section{METHODS}

This study was conducted with 43 mothers of children with cancer in a university hospital. Data were collected by the Beck Anxiety Scale, the Zarit Burden Interview (ZBI), and the Multidimensional Scale of Perceived Social Support.

\section{RESULTS}

The mean age of the mothers was $37.46 \pm 9.67$ old years and the duration of cancer diagnosis was $10.57 \pm 12.59$ months. The primary caregivers of $83.7 \%$ of children were their mothers. It was determined that $76.7 \%$ of mothers had a change in their social life after their children diagnosis. There was a positive correlation between the mothers' Beck Anxiety Scale scores and the ZBI scores ( $r=0.508 ; \mathrm{p}=0.01)$. There was a statistically significant difference between the status of changes in mothers social life and Beck Anxiety Scale $(t=4,454 ; \mathrm{p}<0.001)$ and the $\mathrm{ZBI}$ scores $(\mathrm{t}=3.259 ; \mathrm{p}=0.003)$. The status of mothers receiving support in household chores $(\mathrm{t}=0.864 ; \mathrm{p}=0.018)$ affected their mean scores of $\mathrm{ZBI}$ scores.
\end{abstract}

\section{CONCLUSION}

In this study, as the anxiety level of the mothers increased, the burden of care increased, too. Furthermore, the level of care burden was higher for the mothers who had no support in the household chores and who had a change in social life after the diagnosis. The anxiety level of mothers who reported changes in social life after diagnosis was also high.

Keywords: Anxiety; care burden; child with cancer; social support.

Copyright $\odot$ 2021, Turkish Society for Radiation Oncology

\section{Introduction}

Cancer is considered one of the most important health problems today and the cases are increasing in the world and our country.[1] Childhood cancers are among the most second common causes of death in developed countries, while it is the third cause of mortality in children in Turkey.[2-4] In an international study conducted by the International Association of Cancer Registries (2017), in 62 countries, it was noted that the childhood cancer rate increased $13 \%$ in the period 2001-2010 compared to the 1980s. [5] The 5-year survival rates of children and adolescents diagnosed with cancer in high-income countries were approximately $80-84 \%$, this ratio was $70.8 \%$ for Turkey with the advances in treatment in recent years.[3,6] Treatment 
success and an increase in life expectancy caused family members to actively participate in the care process and take more responsibility.[7]

Being diagnosed with cancer for a child creates a very stressful situation for both children and their parents. [8] Children with cancer require special care and support because of their developmental features. $[9,10]$ In the child and family, stress, and anxiety begin immediately after diagnosis and continues all of the treatments stages. $[9,10]$ It is reported that parents who have children with cancer be more likely to have psychological problems such as anxiety, depression, and stress compared with parents of healthy children.[11,12]

Cancer lead to significant social, physical, economic, and emotional burdens[13] which are characterized by persistent worry, stress, or negative experiences.[14] The burdens for families are categorized as financial needs, work activities, communicating with the siblings of the ill child, dealing with the reactions of others, and concerns about the future of the child. $[15,16]$ Frequent surgeries, medical visits, adverse effects, and the risk of infection all may lead to mothers' psychological distress and increasing their care burden.[16-18] The daily life and routines of the family members who care for the child with cancer are affected seriously and may result in loss of roles in the family members.[19,20] In the literature, it is stated that primer caregivers experience emotional, cognitive, physical, social, and financial problems and these problems increase the burden of caregivers and decrease their quality of life. $[7,18,21]$

The care of the patients is usually carried out by their families, especially mothers, and this is perceived as a family responsibility in Turkey.[22] It was found in a studies that the mothers who played a primary role in treatment experienced more pain, anxiety, and depression than fathers. [23,24] Family members are trying to cope with their complications and also trying to provide support and care for their children with cancer.[9] Children also need their mothers more in the process of treatment and care.[25] For this reason, mothers are often the primary caregivers and most of the disease and the burden of care of the children are placed on mothers.[18] These burnout and difficulties affect both the mothers and the children negatively. $[8,18]$ After a while, mothers experience loss of role, inability to care for their other children, inability to maintain daily life activities, deterioration in the quality of life, and various psychological problems.[26] The child's caregivers experience difficulties and burnouts in the care and treatment process.[18] In this process, nurses and health professionals have important roles for supporting and understanding the effects of caring for children with cancer on caregivers and implementing planned education to reduce these negative emotions.[27] This study was conducted to determine the relationship between the care burden, anxiety, and social support levels of the mothers of the children with cancer, and affecting factors.

\section{Study Questions}

- What is the level of care burden of mothers?

- What is the anxiety level of the mothers?

- What is the level of perceived social support of mothers?

- Is there a relationship between the care burden, anxiety levels, and perceived social support levels of the mothers?

\section{Materials and Methods}

This descriptive study was carried out in a university hospital in Turkey, Pediatric Hematology-Oncology clinic between October 20, 2018, and January 20, 2019. The hospital is the largest university hospital in the Thrace Region and is a 20-bed clinic.

\section{Sample and Recruitment}

The study subjects were mothers of children with cancer who were treated in pediatrics clinics at a university hospital. In the study, Köse et al.,[28] the number of samples was calculated by using the "Beck Anxiety Inventory (BAI)" score average of their study with caregivers. The sample size of the study was determined by a computer program (G-Power 3.1 version). [29] In this program, the required sample number was found to be 51 in the calculation with 0.80 power, $95 \%$ reliability, and 0.60 effect size according to the study by Köse et al. Considering the inclusion and exclusion criteria of the study, the study was completed with 43 mothers (response rate: $84.31 \%$ ) because four mothers were not willing to study and four mothers filled the questionnaires incompletely.

Mothers were eligible for selection if: Their child was diagnosed at least 1 month before the study, (2) chemotherapy or radiotherapy had started, and (3) the mothers were the direct caregivers.

\section{Research Variables}

Independent variables of the research; socio-demographic characteristics of children and parents (age, gender, family structure, employment status, educa- 
tional level, place of residence, number of children, presence of other children with chronic disease, support status about the child care, household chores, etc.); dependent variables are; mean scores of the "Zarit Burden Interview (ZBI)," "BAI," and "Multidimensional Perceived Social Support Scale (MPSSS)."

\section{Data Collection Procedure}

During the data collection period, the researchers interviewed the pediatric oncology clinic staff every day and evaluated them within the scope of the families' inclusion criteria. Mothers who met the sample selection criteria first met the researchers and after a safe relationship was established, the parent was informed about the research. Written permission was obtained from mothers who volunteered to participate in the study. The data were collected in the interview room in the service for mothers to feel comfortable. The data were collected based on the self-report of the mothers through the face-to-face interview method. Data collection took approximately 10-15 min for each participant.

\section{Research Instruments}

The data were collected with "Information Form," "ZBI," "BAI," and "MPSSS."

\section{Information Form}

The form was prepared by the researchers in line with the literature.[7,19,21] The form consists of 22 questions, 15 questions including socio-demographic characteristics of children and parents (age, gender, family structure, employment status, education level, income status, place of residence, number of children, presence of other children with chronic disease, etc.) and seven questions about parents' home care burden, anxiety, and social support status (support status about the child care and household chores, whom they get, etc.).

\section{ZBI}

The scale was developed by Zarit et al.[30] in 1980. Its Turkish validity and reliability study was performed by Inci and Erdem (2008) and the Cronbach alpha value is in the range of $0.87-0.99$.[31] In the current study, the Cronbach's a reliability coefficient of this scale was 0.84 . The scale consists of 22 items to determine the effect of caregiving on the life of the caregiver. Each item is a 4-point Likert-type scale that changes to never (0), rarely (1), sometimes (2), often (3), or almost always (4). The minimum score is " 0 " point and maximum score is " 88 " point; "0-20" point is less burden, " $21-40$ " point is a medium burden, " $41-60$ " point is a high burden, and "61-88" point is an extreme burden.

\section{BAI}

BAI was developed in 1988 by Beck et al.[32] Its Turkish validity and reliability were done by Ulusoy et al.[33] and Cronbach's alpha value was found to be 0.92 . In the current study, the Cronbach's a reliability coefficient of this scale was 0.79 . This scale is a self-report measure of anxiety. BAI which is a Likert-type scale includes a total of 21 somatic and cognitive anxiety symptoms, and the score range is graded between 0 and 3 points, with low, moderate, and high responses. 8-15 points are determined as a low-level anxiety, 16-25 points are moderate-level anxiety, and 26-63 points are determined as score ranges that meet high-level anxiety symptoms. The maximum score that can be obtained from the scale is 63 .

\section{MPSSS}

It was developed by Zimet et al.[34] in 1988 and its validity and reliability study was carried out in 1995 by Eker et al.[35] The scale consists of 12 items in total. The scale is a 7-point Likert-type scale with the form of "Absolutely Yes/No." Three subgroups reflect the support resources of the scale: Family, friend, and private person support, and each subgroup consists of 4 items and the reliability scores are 0.89 on the whole scale and 0.85 on the subgroups. Items $3,4,8$, and 11 in the scale measure family support, $6,7,9$, and 12 items measure friend support, and 1, 2, 5, and 10 items measure private person support. The lowest score that can be obtained from the subscales is 4 , and the highest score is 28. The lowest score that can be obtained from the total scale score obtained by adding the scores obtained from the subscales is 12 , and the highest score is 84 . The scale does not have a cutoff point, and the high score obtained from the scale indicates that perceived social support is also high. In the current study, the Cronbach's a reliability coefficient of this scale was 0.95 .

\section{Ethical Approach}

The Institutional Ethical Committee of the hospital approved the study (approval number: 19/09, date: 19.11.2018). The purpose of the research was explained to the mothers and children before the questionnaire and scales were distributed. Mothers were informed that they do not write names on the form and scales and that the data obtained will be used for only scientific purposes and verbal and written consent was obtained from them. 


\section{Data Analysis}

Statistical Package for the Social Sciences (SPSS) version 20.0 (IBM SPSS Corp.; Armonk, NY, USA) statistical program was used to analyze the data. The sociodemographic characteristics of mothers and children, data on mothers' home care burden, anxiety, and social support status were evaluated by number, percentage, mean, and standard deviation. To examine the normality of the distribution of the data, the KolmogorovSmirnov test was applied. The parametric analysis methods were used for data analysis. The relationships between the socio-demographic characteristics of children and mothers (age, gender, number of children, presence of other children with chronic disease, etc.) and the total and sub-dimensions mean scores of "ZBI," "BAI," and "MPSSS" were analyzed with Student's t-test. Relationships between scale scores were evaluated by Pearson correlation analysis. The results were evaluated at a 95\% confidence interval and $\mathrm{p}<0.05$ significance level.

\section{Results}

The mean age of the mothers was $37.46 \pm 9.67$ years old and the mean age of children was $10.12 \pm 5.28$ years old and $58.1 \%$ of the children were girls. The mean number of children was $2.11 \pm 0.87$ in the family. Children's mean of cancer treatment duration was $10.57 \pm 12.59$ months and $60.5 \%$ of the children were receiving chemotherapy treatment, also $95 \%$ of the children in the remission term. It was found that mothers were primary caregiver of the children (83.7\%), and 55.8\% of mothers received support from their husband for children' care. The mothers' $76.7 \%$ stated that their social life was changed, $20.9 \%$ of them stated that they received support related to household chores, and 34.9\% of them had any disease.

The mean total score of the BAI of the mothers was $20.62 \pm 15.74$ and $37.2 \%$ of mothers had high levels of anxiety. The mean total score of the ZBI of the mothers was $29.83 \pm 20.92$. The mean total score of the MPSSS of the mothers was $56.83 \pm 22.06$, "family" sub-dimension mean score was $20.62 \pm 7.65$, "friend" sub-dimension mean score was $18.69 \pm 8.67$, and "private person" subdimension mean score $17.51 \pm 8.49$ was found (Table 1 ).

There was a significant difference between the mothers' status of change in the social lives and their BAI total score averages $(t=4.454 ; \mathrm{p}<0.001)$ and the mean score of the ZBI $(\mathrm{t}=3.259 ; \mathrm{p}=0.003)$. Mothers who report their social life was changed had higher anxiety levels and caregiver burden (Table 2).
Table 1 Distribution of mothers' $\mathrm{BAI}, \mathrm{ZBI}$, and MPSSS total and sub-dimension mean scores $(n=43)$

\begin{tabular}{lccc} 
Scales and Sub-dimensions & $\mathbf{n}$ & $\%$ & Mean \pm SD \\
\hline BAI & & & $20.62 \pm 15.74$ \\
Anxiety level & & & \\
$\quad$ No anxiety & 11 & 25.6 & \\
$\quad$ Low & 8 & 18.6 & \\
$\quad$ Moderate & 8 & 18.6 & \\
$\quad$ High & 16 & 37.2 & \\
ZBI & & & $29.83 \pm 20.92$ \\
MPSSS & & & $56.83 \pm 22.06$ \\
$\quad$ Family & & & $20.62 \pm 7.65$ \\
$\quad$ Friend & & & $18.69 \pm 8.67$ \\
$\quad$ Private person & & & $17.51 \pm 8.49$ \\
\hline
\end{tabular}

SD: Standard deviation; BAI: Beck anxiety inventory; ZBI: Zarit burden interview; MPSSS: Multidimensional perceived social support scale

Table 2 The comparison of some characteristics of mothers and children with total mean scores and sub-dimension scores of $\mathrm{BAl}, \mathrm{ZBI}$, and MPSSS $(n=43)$

\begin{tabular}{cccc} 
Characteristics & $\begin{array}{c}\text { BAI } \\
\text { Mean } \pm \text { SD }\end{array}$ & $\begin{array}{c}\text { ZBI } \\
\text { Mean } \pm \text { SD }\end{array}$ & $\begin{array}{c}\text { MPSSS } \\
\text { Mean } \pm \text { SD }\end{array}$ \\
\hline Change in social life & & & \\
Yes & $24.06 \pm 16.27$ & $33.87 \pm 21.48$ & $56.03 \pm 22.12$ \\
No & $9.30 \pm 5.43$ & $16.50 \pm 12.02$ & $59.50 \pm 22.81$ \\
$t$ & 4.454 & 3.259 & 0.625 \\
p & $<0.001^{*}$ & $0.003^{*}$ & 0.668 \\
Getting help in & & & \\
household chores & & & \\
Yes & $26.77 \pm 11.15$ & $26.02 \pm 19.74$ & $66.00 \pm 11.96$ \\
No & $19.00 \pm 16.50$ & $44.22 \pm 19.89$ & $54.41 \pm 23.57$ \\
t & 1.330 & 0.864 & 2.040 \\
p & 0.191 & $0.018^{*}$ & 0.052 \\
\hline
\end{tabular}

${ }^{*} \mathrm{p}<0.05$ (statistically significant). SD: Standard deviation; t: Mann Whitney U test; BAl: Beck anxiety inventory; ZBI: Zarit burden interview; MPSSS: Multidimensional perceived social support scale

There was a significant difference between the mothers' getting help at household chores and their total mean scores of the ZBI $(\mathrm{t}=0.864 ; \mathrm{p}=0.018)$. The caregiving burden of mothers who did not receive help at household chores was higher (Table 2).

A moderate positive correlation was found between the total scores of BAI and ZBI of mothers $(r=0.508$; $\mathrm{p}=0.001)$. As mothers' anxiety increased, caregiver burden also increased (Table 3 ).

There was no statistically significant correlation between the ZBI and MPSSS scores of mothers. Furthermore, there was no statistically significant correlation 
Table 3 The relationship between total and sub-dimension scores of $\mathrm{ZBI}, \mathrm{BAI}$ and MPSSS of mothers $(n=43)$

\begin{tabular}{cccccc} 
Scales & $\begin{array}{c}\text { ZBI } \\
\text { total }\end{array}$ & $\begin{array}{c}\text { MPSSS } \\
\text { family }\end{array}$ & $\begin{array}{c}\text { MPSSS } \\
\text { friend }\end{array}$ & $\begin{array}{c}\text { MPSSS } \\
\text { private } \\
\text { person }\end{array}$ & $\begin{array}{c}\text { MPSSS } \\
\text { total }\end{array}$ \\
\hline $\begin{array}{c}\text { BAl total } \\
\text { r }\end{array}$ & 0.508 & 0.150 & 0.071 & 0.034 & 0.093 \\
p & $0.001^{*}$ & 0.338 & 0.650 & 0.829 & 0.554 \\
ZBI & & & & & \\
r & - & -0.022 & -0.068 & 0.161 & 0.028 \\
p & - & 0.890 & 0.664 & 0.303 & 0.861 \\
$\begin{array}{c}\text { Duration of } \\
\text { diagnosis }\end{array}$ & & & & & \\
$\quad$ r & -0.029 & 0.116 & 0.149 & 0.090 & 0.132 \\
p & 0.858 & 0.463 & 0.347 & 0.569 & 0.405 \\
\hline
\end{tabular}

${ }^{*} p<0.05$ (statistically significant). r: Pearson correlation analysis; BAl: Beck anxiety inventory; ZBI: Zarit burden interview; MPSSS: Multidimensional perceived social support scale

between the duration of cancer treatment of the children and total mean scores of ZBI, MPSSS, and BAI ( $>0.05)$ (Table 3).

\section{Discussion}

Children with cancer need more mothers to meet their needs and take care of them during the cancer treatment process.[36] Therefore, it is the mother who experiences more challenges and who is most affected by the treatment phase and as well as the cancer care process for the child. [37] In the literature, the care burden and psychosocial problems of pediatric oncology patients' mothers are generally examined as descriptive and qualitative studies.[37-39] In the literature, the care burden, psychosocial problems, and support system experienced by mothers who had children with cancer were examined separately.[16,17,36] There are limitations in this studies that explain how these variables affect each other. Therefore, this study was conducted to determine the relationship between the care burden, anxiety, and social support levels of the mothers of children with cancer and affecting factors.

The incidence of childhood cancers is increasing rapidly in the world.[28] The long course of treatment affects both families and children negatively. [8] In Turkey, it is usually allowed the mothers to stay with their children in the oncology clinic. As primary caregivers are mothers so, their anxiety, stress, anxiety, care burden are more than other fam- ily members.[36] In this study, it was determined that $37.2 \%$ of mothers had high anxiety level, the mean total score of the ZBI, and the MPSSS of the mothers showed that mother's had moderate care burden, and moderate perceived social support, and mothers mostly receive social support from their families (Table 1). In the literature, similar to these findings, it is seen that the level of social support of the mother is moderate and they received social support mostly from their families. $[9,28,40]$ In a study conducted by Baran, the mothers who had children with acute lymphoblastic leukemia (ALL) had high care burden.[18] van Warmerdam et al.[7] conduct a meta-analysis to determine the prevalence of psychological problems in parents children with cancer and they found that parents of the children with cancer show a greater incidence of anxiety. It can be said that childhood cancer not only affects children but also all the family members: Parents, well siblings, and others members.[41] According to the research results, primer caregiver especially mothers experiences more stress, anxiety, and care burden than other family members. $[16-18,42]$ It is very important for mothers need to be supported by their families and health professionals to reduce the care burden and negative feelings that they experience.[43] Health professionals who spend the most time with mothers in hospitals are nurses. Nurses have important roles in guiding mothers to cope with their negative emotions. [44] The literature suggests that nurses plan the care of the child together with their parents, creating environments where parents will feel comfortable, informing families during and after treatment, and actively involve the family in the entire care of the child.[44-46] These nursing interventions should be implemented for reducing the mother's anxiety and care burden's level.

One of the important results of this study is that mothers who report changes in social life had a high score of the anxiety and care burden (Table 2). This result is consistent of the literature. Chiou et al.[47] found that cancer caregivers who received social support had a low level of care burden and the care burden decreased as perceived social support increased. The child being diagnosed with cancer does not only change the life of the child but also the routine life and responsibilities of caregivers.[15] The mothers are often depressed and isolated because of the treatment and the care burden of the disease and therefore restrict relationships within their environments. [17] These problems may continue for a few years.[17] According to the Theory of Human Ecology, people's environment 
and social supports play an important role in their responses to stressors. [48] According to this theory, if the mothers of children with cancer are socially supported, their psychological problems and care burdens can be reduced.[17] Furthermore, social interaction is a cultural component that has the effect of reducing parents' distress, care burden, and anxiety.[17]

In a recent study, it was found that the care burden of mothers who did not receive help at chores was high (Table 2). This finding consisted of the literature. Wang et al.[40] explored that care burden among parents of children with ALL and it was found that parents with low care burden tend to have more time for daily care, more co-caregivers, higher incomes, less depression, better emotional well-being, and social support. Caregivers are usually responsible for about $80 \%$ of the duties that were performed by health professionals (e.g., side effects control, and late medication effects), in addition to their personal responsibilities.[15] In this process, when mothers cannot get help from anyone, the burden of care increases. [21] In the literature, it was reported that many of the treatments are provided in the outpatient setting, minimizing hospitalization, but this situation increases the care burden of caregivers, especially mothers, at home.[41] Other family members in the home can assist in the care, otherwise, as indicated in the research results, the care burden of mothers may increase and this may have other consequences.

Childhood cancers creates a vulnerability of psychosocial problems for both parents and children, including a unique risk of decreasing quality of life. [49] Since the diagnosis of cancer, caregivers have experienced fear, anxiety, insomnia, and post-traumatic stress. [8,36,49] Providing emotional and physical care to the child not only raises the care burden of the mother, but it may also lead to their physical well-being to worsen.[36] Consistent with the literature, we found that when mothers' anxiety increased, the their care burden also increased (Table 3). Köse et al.[28] analyzed the relationship between the care burden and anxiety levels of parents whose child has cancer and they stated that positive correlation between the care burden and anxiety levels of parents. Furthermore, Santo et al.[16] determined the care burden and quality of life of children's caregivers, it was reported that the negative correlation between care burden and mental well-being. It should be remembered that cancer is a major challenge for both children and families and causes high levels of anxiety.[9] Nurses should support both the child and the family in all processes beginning from the diagnosis.[44] Swanson's theory of care suggests that the nurse, patient, and parent relationship is essential for care, and the implementation of initiatives that "promote, restore, and maintain optimal health for individuals."[50] Therefore, nurses should build a relationship with parents to meet parent's needs.

\section{Limitations of the Study}

This study has some limitations. The small sample size is limited by the number of mothers with cancer children being treated who may not be representative of the general population. Furthermore, the present study did not examine possible effects of treatment intensity (i.e., radiation dose and chemotherapy protocol).

\section{Conclusion}

As a consequence of the present study, care burden and perceived social support levels by Turkish mothers of children with cancer were moderate, but $37.2 \%$ of them high level of anxiety. It was found that mothers received the most support from their "families." Mothers who reported social life changes had a higher level of anxiety and care burden. One of the important results of the study is that it was determined a correlation between the mother's anxiety score and mother's care burden score. In the light of all these findings, mothers should be included in the care of the child by the nurses and they should inform mothers about diseases, treatments, and all interventions to reduce the negative effect of the care. Nurses should build relationships with family members from the beginning of the diagnosis. Besides, nurses should assess both children and mother's concerns and psychological needs. Based on the assessment outcomes, programs, and activities such as parental training and psychological counseling can implemented for mothers.

Note: This study was presented at the $2^{\text {nd }}$ International $7^{\text {th }}$ National Pediatric Nursing Congress as a verbal presentation (27-30 November 2019/Izmir, Turkey).

Acknowledgement: Thank you so much to the mothers for the information that they agreed to participate in the research.

Peer-review: Externally peer-reviewed.

Conflict of Interest: All authors declared no conflict of interest.

Ethics Committee Approval: The study was approved by the Trakya University Faculty of Medicine Scientific Research Ethics Committee (No: 19/09, Date: 19/11/2018). 
Financial Support: None declared.

Authorship contributions: Concept - E.N.K., M.A.K., R.S., İ.Ç.; Design - E.N.K., R.S., M.A.K., İ.Ç.; Supervision E.N.K., M.A.K.; Funding - None; Materials - E.N.K., R.S.; Data collection and/or processing - E.N.K., R.S.; Data analysis and/or interpretation - E.N.K., R.S.; Literature search E.N.K., R.S., İ.Ç.; Writing - E.N.K., R.S., M.A.K., İ.Ç.; Critical review - E.N.K., M.A.K., İ.Ç., R.S.

\section{References}

1. Demir Küreci H, Örnek Büken N. Evaluation of involvement of children/adolescents diagnosed with cancer in their own treatment and assent process. J Curr Pediatr 2016;14(1):37-45.

2. Kırzıoğlu Z, Güney G. Multidisciplinary approaches in childhood cancers. Turk Klin Pediatr Dent Spec Top 2019;5(1):1-6.

3. Kutluk MT, Yeşilipek A. Pediatric Cancer Registry in Turkey 2009-2018 (TPOG and TPHD). J Clin Oncol 2019;37(15):e21510.

4. American Cancer Society. Key statistics for childhood cancers: The American Cancer Society Medical and Editorial Content Team; 2020. Available at: https:// www.cancer.org/cancer/cancer-in-children/key-statistics.html. Accessed Aug 24, 2020.

5. Steliarova-Foucher E, Colombet M, Ries LA, Moreno F, Dolya A, Bray F, et al. International incidence of childhood cancer, 2001-10: A population-based registry study. Lancet Oncol 2017;18(6):719-31.

6. Bhakta N, Force LM, Allemani C, Atun R, Bray F, Coleman MP, et al. Childhood cancer burden: A review of global estimates. Lancet Oncol 2019;20(1):e42-53.

7. van Warmerdam J, Zabih V, Kurdyak P, Sutradhar R, Nathan PC, Gupta S. Prevalence of anxiety, depression, and posttraumatic stress disorder in parents of children with cancer: A meta-analysis. Pediatr Blood Cancer 2019;66(6):e27677.

8. Peterson RK, Chung J, Barrera M. Emotional symptoms and family functioning in caregivers of children with newly diagnosed leukemia/lymphomas and solid tumors: Short-term changes and related demographic factors. Pediatr Blood Cancer. 2020;67(2):e28059.

9. Götze H, Friedrich M, Brähler E, Romer G, Mehnert A, Ernst J. Psychological distress of cancer patients with children under 18 years and their partners a longitudinal study of family relationships using dyadic data analysis. Support Care Cancer 2017;25(1):255-64.

10. Hassan GA, Ibrahim HS. The effect of supportive nursing intervention on the burden and coping strategies of caregivers of children with cancer. J Nurs Educ Pract 2018;8(7):125.

11. Rahmani A, Azadi A, Pakpour V, Faghani S, Afsari
EA. Anxiety and depression: A cross-sectional survey among parents of children with cancer. Indian J Palliat Care. 2018;24(1):82-5.

12. Shin J, Lim JW, Shin D, Kim S, Yang HK, Cho J, et al. Underestimated caregiver burden by cancer patients and its association with quality of life, depression and anxiety among caregivers. Eur J Cancer Care 2018;27(2):e12814.

13. International Agency for the Research of Cancer. World Cancer Report: Cancer Research For Cancer Prevention. London: International Agency for the Research of Cancer; 2014.

14. Govina O, Vlachou E, Kalemikerakis I, Papageorgiou D, Kavga A, Konstantinidis T. Factors associated with anxiety and depression among family caregivers of patients undergoing palliative radiotherapy. Asia Pac J Oncol Nurs 2019;6(3):283-91.

15. Cooper DL, Powe BD, Smith T. Social support provided by and strain experienced by AfricanAmerican cancer caregivers. Support Care Cancer 2013;21(10):2719-25.

16. Santo EARdE, Gaíva MAM, Espinosa MM, Barbosa DA, Belasco AG. Taking care of children with cancer: Evaluation of the caregivers' burden and quality of life. Rev Lat Am Enfermagem 2011;19(3):515-22.

17. Harper FW, Peterson AM, Albrecht TL, Taub JW, Phipps S, Penner LA. Satisfaction with support versus size of network: Differential effects of social support on psychological distress in parents of pediatric cancer patients. Psychooncology 2016;25(5):551-8.

18. Baran G. The burden of care and life satisfaction of the Turkish mothers of children with cancer. J Client Cent Nurs Care 2018;4(4):175-84.

19. Deshields TL, Rihanek A, Potter P, Zhang Q, Kuhrik M, Kuhrik N, et al. Psychosocial aspects of caregiving: Perceptions of cancer patients and family caregivers. Support Care Cancer 2012;20(2):349-56.

20. Yildız MK, Ekinci M. The relation between anger expression styles and caretaking burden of family members of cancer patients and affecting factors. J Educ Res Nurs 2017;14(3):176-84.

21. Yildiz E, Dedeli O, Pakyuz CS. Evaluation of care burden and quality of life among family caregivers of patients with cancer. J Educ Res Nurs 2016;13(3):216-25.

22. Orak OS, Sezgin S. Kanser hastasına bakım veren aile bireylerinin bakım verme yüklerinin belirlenmesi. J Psychiatr Nurs. 2015;6(1):33-9.

23. Ghufran M, Andrades M, Nanji K. Frequency and severity of depression among mothers of children with cancer: Results from a teaching hospital in Karachi, Pakistan. Br J Med Pract 2014;7(1):a701.

24. Al-Maliki SK, Al-Asadi J, Al-Waely A, Agha S. Prevalence and levels of depression among parents of children with cancer in Basrah, Iraq. Sultan Qaboos Univ 
Med J 2016;16(3):e329.

25. Wijnberg-Williams BJ, Kamps WA, Klip EC, Hoekstra-Weebers JE. Psychological adjustment of parents of pediatric cancer patients revisited: Five years later. Psychooncology 2006;15(1):1-8.

26. Clarke NE, McCarthy MC, Downie P, Ashley DM, Anderson VA. Gender differences in the psychosocial experience of parents of children with cancer: A review of the literature. Psychooncology 2009;18(9):907-15.

27. Jones B, Currin-Mcculloch J, Pelletier W, Sardi-Brown V, Brown P, Wiener L. Psychosocial standards of care for children with cancer and their families: A national survey of pediatric oncology social workers. Soc Work Health Care 2018;57(4):221-49.

28. Köse S, Arar AT, Yıldırım G. The relationship between the care loads and anxiety levels of parents whose child has cancer. J Educ Res Nurs 2019;16(4):282-7.

29. Faul F, Erdfelder E, Lang AG, Buchner A. G* power 3: A flexible statistical power analysis program for the social, behavioral, and biomedical sciences. Behav Res Methods 2007;39(2):175-91.

30.Zarit SH, Reever KE, Bach-Peterson J. Relatives of the impaired elderly: Correlates of feelings of burden. Gerontologist 1980;20(6):649-55.

31. Inci FH, Erdem M. Validity and reliability of the burden interview and its adaptation to Turkish. J Ataturk Univ Sch Nurs 2008;11:85-97.

32. Beck AT, Epstein N, Brown G, Steer R. An inventory for measuring clinical anxiety: Psychometric properties. J Consult Clin Psychol 1988;56(6):893-7.

33. Ulusoy M, Sahin N, Erkmen H. Turkish version of the beck anxiety inventory: Psychometric properties. J Cogn Psychother. 1998;12(2):163-72.

34. Zimet GD, Dahlem NW, Zimet SG, Farley GK. The multidimensional scale of perceived social support. J Pers Assess 1988;52(1):30-41.

35. Eker D, Arkar H, Yaldı H. Factorial structure, validity, and reliability of revised form of the multidimensional scale of perceived social support. Turk Psikiyatr Derg. 2001;12(1):17-25.

36. Bhattacharya K, Pal S, Acharyya R, Dasgupta G, Guha P, Datta A. Depression and anxiety in mothers of children with cancer and how they cope with it: A crosssectional study in Eastern India. Asian J Psychiatr 2016;17:1-8.

37. Baran G, Sürücü HA, Üzel VH. Resilience, life satisfaction, care burden and social support of mothers with a child with acute lymphoblastic leukaemia: A comparative study. Scand J Caring Sci 2020;34(2):340-7.
38. Hatami F, Hojjati H. Effect of roy's adaptation model on the care burden of mothers of children under chemotherapy (a quasi-experimental study). Med Surg Nurs J 2019;8(1):e90489.

39. Arab M, Bernstein C, Haghshenas A, Ranjbar H. Factors associated with caregiver burden for mothers of children undergoing acute lymphocytic leukemia (ALL) treatment. Palliat Support Care 2020;18(4):405-12.

40. Wang J, Shen N, Zhang X, Shen M, Xie A, Howell D, et al. Care burden and its predictive factors in parents of newly diagnosed children with acute lymphoblastic leukemia in academic hospitals in China. Support Care Cancer 2017;25(12):3703-13.

41. Williams PD, Williams KA, Williams AR. Parental caregiving of children with cancer and family impact, economic burden: Nursing perspectives. Issues Compr Pediatr Nurs 2014;37(1):39-60.

42. Klassen AF, Anthony SJ, Khan A, Sung L, Klaassen R. Identifying determinants of quality of life of children with cancer and childhood cancer survivors: A systematic review. Support Care Cancer 2011;19(9):1275-87.

43. Woźniak K, Iżycki D. Cancer: A family at risk. Prz Menopauzalny 2014;13(4):253-61.

44. Enskär K, Darcy L, Björk M, Knutsson S, Huus K. Experiences of young children with cancer and their parents with nurses' caring practices during the cancer trajectory. J Pediatr Oncol Nurs 2020;37(1):21-34.

45. Enskär K, Björk M, Knutsson S, Granlund M, Darcy L, Huus K. A Swedish perspective on nursing and psychosocial research in paediatric oncology: A literature review. Eur J Oncol Nurs 2015;19(3):310-7.

46. Darcy L, Björk M, Knutsson S, Granlund M, Enskär K. Following young children's health and functioning in everyday life through their cancer trajectory. J Pediatr Oncol Nurs 2016;33(3):173-89.

47. Chiou CJ, Chang HY, Chen IP, Wang HH. Social support and caregiving circumstances as predictors of caregiver burden in Taiwan. Arch Gerontol Geriatr 2009;48(3):419-24.

48. Bronfenbrenner U. The Ecology of Human Development. Cambridge, MA: Harvard University Press; 1979.

49. Bakula DM, Sharkey CM, Perez MN, Espeleta HC, Gamwell KL, Baudino M, et al. The relationship between parent distress and child quality of life in pediatric cancer: A meta-analysis. J Pediatr Nurs 2020;50:14-9.

50. Swanson KM. Nursing as informed caring for the wellbeing of others. Image J Nurs Sch 1993;25(4):352-7. 